\section{AB1082 INFLUENCE OF THE VARIATION OF THE OPERATOR, PATIENT POSITION AND DEVICE ON THE MEASUREMENT PERFORMANCE OF RADIOFREQUENCY ECHOGRAPHIC MULTI SPECTROMETRY (REMS)}

C. Caffarelli ${ }^{1}$, G. Adami ${ }^{2}$, G. Arioli ${ }^{3}$, G. Bianchi ${ }^{4}$, M. L. Brandi ${ }^{5}$, S. Casciaro ${ }^{6}$, L. Cianferotti ${ }^{5}$, D. Ciardo ${ }^{6}$, F. Conversano ${ }^{6}$, D. Gatti ${ }^{2}$, G. Girasole ${ }^{4}$, M. Manfedini ${ }^{3}$, M. Muratore ${ }^{7}$, P. Pisani ${ }^{6}$, E. Quarta ${ }^{7}$, L. Quarta ${ }^{7}$, S. Gonnelli ${ }^{1}$ ${ }^{1}$ University of Siena, Department of Medicine, Surgery and Neurosciences, Siena, Italy; ${ }^{2}$ University of Verona, Rheumatology Unit, Department of Medicine, Verona, Italy; ${ }^{3}$ Carlo Poma Hospital, ASST-Mantova, Department of Neurosciences and Rehabilitation, Mantova, Italy; ${ }^{4}$ ASL 3 Genovese, Genoa, Italy; ${ }^{5}$ University Hospital of Florence, Department of Experimental and Clinical Biomedical Sciences, Metabolic Bone Diseases Unit, Florence, Italy; ${ }^{6}$ National Research Council, Institute of Clinical Physiology, Lecce, Italy; ${ }^{7}$ Galateo Hospital, ASL-LE, O.U. of Rheumatology, San Cesario di Lecce, Italy

Background: The monitoring of bone mineral density (BMD) is a key aspect for patients undergoing pharmacological treatments that might cause BMD changes at non-physiological rates. At present, the short-term follow-up of patients under treatment in terms of BMD change with time remains an unmet clinical need, since the current techniques, including the gold standard dual X-ray absorptiometry (DXA), require at least 1 year between two consecutive measurements [1]. Therefore, an effective strategy for the assessment of BMD should guarantee high accuracy, precision and repeatability of the measurements.

Objectives: The aim is to assess the influence of the variation 1) in patient position, 2) operator (both intra- and inter-) and 3) device on the REMS performance at lumbar spine and femoral neck.

Methods: 210 women were enrolled, divided in 7 groups of 30-patient each for the assessment of the parameters of interest, i.e. inter-device, intra- and inter-operator repeatability for lumbar spine scans and inter-patient position, inter-device, intra- and inter-operator repeatability for femoral neck scans.

All patients underwent 2 REMS scans at lumbar spine or femoral neck, performed by the same operator or by 2 different operators or by the same operator using 2 different devices or in different patient position (i.e. supine without constraints or with a constrained $25^{\circ}$-rotation of the leg). The percentage coefficient of variation (CV\%) with $95 \%$ confidence interval and least significant change for a 95\% confidence level (LSC) have been calculated.

Results: For lumbar spine, intra-operator repeatability resulted in $\mathrm{CV} \%=0.37 \%$ (95\%Cl: $0.26 \%-0.48 \%)$, with $\mathrm{LSC}=1.02 \%$, inter-operator repeatability resulted in CV\% $=0.55 \%(95 \% \mathrm{Cl}: 0.42 \%-0.68 \%)$, with $\mathrm{LSC}=1.52 \%$, inter-device repeatability resulted in CV\%=0.53\% (95\% Cl: $0.40 \%-0.66 \%$ ), with LSC $=1.47 \%$.

For femoral neck, intra-operator repeatability resulted in $\mathrm{CV} \%=0.33 \%(95 \% \mathrm{Cl}$ : $0.23 \%-0.43 \%$ ), with $\mathrm{LSC}=0.91 \%$, inter-operator repeatability resulted in CV\% $=0.47 \%$ (95\% Cl: $0.35 \%-0.59 \%)$, with $\mathrm{LSC}=1.30 \%$, inter-device repeatability resulted in $\mathrm{CV} \%=0.42 \%$ (95\% Cl: $0.30 \%-0.51 \%)$, with LSC $=1.16 \%$, inter-patient position repeatability resulted in $\mathrm{CV} \%=0.24 \%(95 \% \mathrm{Cl}: 0.18 \%-0.30 \%)$, with LSC $=0.66 \%$.

Conclusion: REMS densitometry is highly precise for both anatomical sites, showing high performance in repeatability. These results suggest that REMS might be a suitable technology for short-term monitoring. Moreover, thanks to its ionizing radiation-free approach, it might be applied for population mass investigations and prevention programs also in paediatric patients and pregnant women.

References: Note: Carla Caffarelli, Giovanni Adami ${ }^{\S}$, Giovanni Arioli ${ }^{\S}$, Gerolamo Bianchi ${ }^{\S}$, Maria Luisa Brandi $i^{\S}$, Sergio Casciaro ${ }^{\S}$, Luisella Cianferotti ${ }^{\S}$, Delia Ciardo $^{\S}$, Francesco Conversano ${ }^{\S}$, Davide Gatti ${ }^{\S}$, Giuseppe Girasole ${ }^{\S}$, Monica Manfredini ${ }^{\S}$, Maurizio Muratore ${ }^{\S}$, Paola Pisani ${ }^{\S}$, Eugenio Quarta ${ }^{\S}$, Laura Quarta ${ }^{\S}$, Stefano Gonnelli

$\S$ Equal contributors listed in alphabetical order

Disclosure of Interests: Carla Caffarelli: None declared, Giovanni Adami: None declared, Giovanni Arioli *: None declared, Gerolamo Bianchi Grant/research support from: Celgene, Consultant of: Amgen, Janssen, Merck Sharp \& Dohme, Novartis, UCB, Speakers bureau: Abbvie, Abiogen, Alfa-Sigma, Amgen, BMS, Celgene, Chiesi, Eli Lilly, GSK, Janssen, Medac, Merck Sharp \& Dohme, Novartis, Pfizer, Roche, Sanofi Genzyme, Servier, UCB, Maria Luisa Brandi: None declared, Sergio Casciaro: None declared, Luisella Cianferotti: None declared, Delia Ciardo: None declared, Francesco Conversano: None declared, Davide Gatti Speakers bureau: Davide Gatti reports personal fees from Abiogen, Amgen, Janssen-Cilag, Mundipharma, outside the submitted work., Giuseppe Girasole: None declared, Monica Manfedini: None declared, Maurizio Muratore: None declared, Paola Pisani: None declared, Eugenio Quarta: None declared, Laura Quarta: None declared, Stefano Gonnelli: None declared

DOI: 10.1136/annrheumdis-2020-eular.4553

\section{AB1083 \\ CURRENT PRACTICE AND OPINIONS ON IMAGING- GUIDED INTERVENTIONAL PROCEDURES IN RHEUMATIC AND MUSCULOSKELETAL DISEASES: INTERIM RESULTS OF A MULTINATIONAL MULTIDISCIPLINARY SURVEY TO INFORM EULAR POINTS TO CONSIDER}

F. Carubbi ${ }^{1}$, P. Bosch', P. M. Machado ${ }^{3}$, C. A. Scirè ${ }^{4}$, A. Alunno ${ }^{5}$, X. Baraliakos ${ }^{6}$, C. Dejaco ${ }^{2} .{ }^{1}$ Rheumatology Unit, L'Aquila, Italy; ${ }^{2}$ Medical University Graz, Graz, Austria; ${ }^{3} \mathrm{UCL}$, London, United Kingdom; ${ }^{4}$ University of Ferrara, Ferrara, Italy; ${ }^{5}$ Rheumatology Unit, Perugia, Italy; ${ }^{6}$ Ruhr University Bochum, Bochum, Germany

Background: maging is widely used for diagnostic purposes in patients with rheumatic and musculoskeletal diseases (RMDs). In recent years, it is increasingly used also to guide interventional procedures. However, the extent of imaging application for this purpose as well as the different technical standards employed across Europe are not known.

Objectives: To learn how much imaging is used for interventional procedures in RMDs. To explore the technical standards employed in different settings and how important they are rated by users.

Methods: As part of the work of a multidisciplinary EULAR Task Force to develop recommendations for the use of imaging to guide interventional procedures in patients with RMDs, a survey was developed. The survey explored aspects of different interventional procedures (e.g. joint aspiration/injection) such as the use of imaging guide and the technical standards. Respondents provided also a $0-10$ rating of how important they considered the same conditions/items with regard to each procedure. The survey was distributed to: rheumatologists across Europe, USA, Central America, South America, Asia and Pacific Area, HPs across Europe, European and American associations of other specialities (e.g. radiology, anaesthesiology). The survey was launched in December 2019. Interim results after 4 weeks are presented.

Results: 200 responses from 36 countries were collected. The respondents were mainly rheumatologists (90\%) (Figure 1). $90 \%$ of respondents performed interventional procedures related to RMDs and of these, $76 \%$ use imaging guide. Ultrasonography (US) is the most commonly used technique (96\%) followed by X-ray/fluoroscopy (13\%). Among respondents using imaging guide, $60 \%$ received training on both imaging and imaging-guided procedures, $20 \%$ only on imaging and $16 \%$ no training. $49 \%$ of respondents perform the whole procedure using direct image guidance, $21 \%$ use imaging to find the appropriate anatomical landmark and then perform the procedure blindly. Air and contrast agent to control needle placement are rarely used $(\leq 20 \%)$. Respondents provided also a rating (0-10) of how important they considered different technical conditions/items for each procedure and an estimate on a Likert scale of how often they used them for each of the procedures (Figure 1 shows an example). In most cases respondents use always/most of the times the conditions/items that they considered important. Discrepancies were mainly due to barriers at their own center.

Conclusion: Imaging, mainly US, is widely used to guide interventional procedures. However, training is not homogeneous and the use of imaging guide as well as technical conditions are based on the operator's opinion/experience. This survey will inform the EULAR points to consider for the use of imaging to guide interventional procedures in patients with RMDs.

Table 1. Characteristics of respondents $(n=200)$

\begin{tabular}{lcc}
\hline & N & $\%$ \\
\hline Age & & \\
$\leq 30$ & 38 & 19 \\
$31-35$ & 56 & 28 \\
$36-39$ & 50 & 25 \\
$40-49$ & 33 & 16.5 \\
$\geq 50$ & 23 & 11.5 \\
Gender & & \\
Female & 89 & 44.5 \\
Male & 111 & 55.5 \\
Specialty/Position & & 90 \\
Rheumatology & 180 & 5 \\
Radiology & 11 & 1.5 \\
Physical medicine and rehabilitation & 3 & 2 \\
Pediatrics & 4 & 1.5 \\
Non-linical researcher & 3 & 1.5 \\
Health professionals & 3 & 1.5 \\
Other & 3 & \\
& &
\end{tabular}

Disclosure of Interests: Francesco Carubbi Speakers bureau: Francesco Carubbi received speaker honoraria from Abbvie and Celgene outside this work., Philipp Bosch: None declared, Pedro M Machado Consultant of: PMM: Abbvie, Celgene, Janssen, Lilly, MSD, Novartis, Pfizer, Roche and UCB, Speakers bureau: PMM: Abbvie, BMS, Lilly, MSD, Novartis, Pfizer, Roche and UCB, 
Carlo Alberto Scirè: None declared, Alessia Alunno: None declared, Xenofon Baraliakos Grant/research support from: Grant/research support from: AbbVie, BMS, Celgene, Chugai, Merck, Novartis, Pfizer, UCB and Werfen, Consultant of: AbbVie, BMS, Celgene, Chugai, Merck, Novartis, Pfizer, UCB and Werfen, Speakers bureau: AbbVie, BMS, Celgene, Chugai, Merck, Novartis, Pfizer, UCB and Werfen, Christian Dejaco: None declared DOI: 10.1136/annrheumdis-2020-eular.6070

\section{AB1084 $\quad$ A SYSTEMATIC REVIEW OF THE ABILITY OF WHOLE BODY MRI TO ASSESS DISEASE ACTIVITY AND TREATMENT RESPONSE IN INFLAMMATORY ARTHRITIS}

V. Choida ${ }^{1,2}$, A. V. Madenidou ${ }^{1,2}$, D. Sen ${ }^{1,2}$, M. Hall-Craggs ${ }^{3,4}$, C. Ciurtin ${ }^{1,2}$ ${ }^{1}$ University College London, Centre for Adolescent Rheumatology Versus Arthritis, London, United Kingdom; ${ }^{2}$ University College London Hospitals NHS Foundation Trust, Rheumatology Department, London, United Kingdom; ${ }^{3}$ University College London Hospitals NHS Foundation Trust, Radiology Department, London, United Kingdom; ${ }^{4}$ University College London, Centre for Medical Imaging, London, United Kingdom

Background: Whole body MRI (WBMRI) is an imaging technique that allows the assessment of the spine and peripheral joints in patients with inflammatory arthritis (IA) in a single examination. Depending on the protocol, it can potentially identify synovitis, enthesitis, spondyloarthritis and chronic structural changes. Objectives: To evaluate the performance of WBMRI in patients with IA for detecting inflammation compared with clinical assessments and to show changes in response to treatment.

Methods: We conducted a systematic search of the electronic databases MEDLINE, EMBASE and Cochrane Library. Two authors selected independently the eligible studies, extracted the predefined data and assessed the quality using the QUADAS2 tool. Studies that reported a)disease activity scores, b)patient or physician reported outcomes or c)results from other imaging tests in IA patients who underwent WBMRI were included.

Results: Fourteen studies out of $\mathbf{4 7 1}$ met our inclusion criteria. The majority of the studies were performed in Spondyloarthritis [SpA] $(n=9)$, followed by Rheumatoid Arthritis [RA] $(n=4)$ and Psoriatic Arthritis [PsA] patients $(n=3)$. Nine studies provided clinical and MRI outcome measures. There was great heterogeneity in the quality of studies, disease specific outcomes reported and methodology used to compare with MRI findings. One study documented low correlation between 28 swollen/tender joint count and MRI bone marrow oedema (BME)/ synovitis in RA patients, whereas another reported that $31 \%$ of $M R I$ negative joints (other than hand joints) exhibited tenderness. In PsA, one study demonstrated correlation between 28 swollen joint count and BME $(r=0.54, p=0.03)$. Superiority of WBMRI in the detection of synovitis and enthesitis over clinical examination was documented in two studies with SpA patients. A third study in SpA showed a ranging agreement of 49 to $100 \%$ between clinical and WBMRI enthesitis.

Treatment response to biologics was assessed by WBMRI in 7 studies ( 5 in SpA, 2 in RA). In RA, one study showed numerical (but not statistically significant) reduction of WBMRI joint count at week 16 and 52 of Adalimumab treatment, whereas the reduction was statistically significant for the subset of patients achieving good EULAR response at week 16 . The other study demonstrated a reduction in WBMRI synovitis and bone oedema scores after 1 year of anti-TNF or Tocilizumab treatment (median DAS28 score decreased from 5.1 to 2.1). A multicentre open label study reported a reduction in the number of MRI enthesitis lesions, spinal and sacroiliac joint scores at week 48, year 2 and 3 of Etanercept treatment in SpA patients. The mean BASDAI score decreased from 5.4 at baseline to 1.5 at year 2 and 2.2 at year 3. Improvement in WBMRI scores in SpA was also documented in one Adalimumab and one further Etanercept study.

Conclusion: There was a variable level of correlation between clinical and WBMRI outcome measures across the included studies. The clinical significance of inflammation detected by WBMRI in some studies remains unclear. Many of the devised WBMRI scores appear to decrease after biologic treatment. Further studies are needed to determine the accuracy of WBMRI in detecting inflammation and its potential utility for clinical practice.

Disclosure of Interests: Varvara Choida: None declared, Anastasia-Vasiliki Madenidou: None declared, Debajit Sen: None declared, Margaret Hall-Craggs: None declared, Coziana Ciurtin Grant/research support from: Pfizer, Consultant of: Roche, Modern Biosciences

DOI: 10.1136/annrheumdis-2020-eular.2576

\section{AB1085 \\ ASSESSMENT OF DIAGNOSTIC DELAY IN PATIENTS AFFECTED BY ENTEROPATHIC SPONDYLOARTHRITIS: A CROSS-SECTIONAL STUDY}

P. Conigliaro $^{1}$, M. S. Chimenti ${ }^{1}$, A. D'antonio ${ }^{1}$, A. Wlderk ${ }^{2}$, L. Sichi ${ }^{1}$,

P. Triggianese ${ }^{1}$, F. Sabuzi ${ }^{2}$, G. Sena ${ }^{3}$, N. Alfieri ${ }^{3}$, V. Da Ros ${ }^{2}$, L. Biancone ${ }^{3}$,
R. Perricone ${ }^{1} .{ }^{1}$ University of Rome Tor Vergata, Rheumatology, Allergology and Clinical Immunology, Department of "Medicina dei Sistemi", Rome, Italy; ${ }^{2}$ University of Rome Tor Vergata, Department of Diagnostic Imaging and Interventional Radiology, Rome, Italy; ${ }^{3}$ University of Rome Tor Vergata, Gastroenterology Unit, Department of “Medicina dei Sistemi", Rome, Italy

Background: Diagnostic delay of spondyloarthritis ( $\mathrm{SpA}$ ) has been established even in combination with inflammatory bowel disease and may contribute to radiographic progression and disability.

Objectives: We aimed to evaluate diagnostic delay in enteropathic SpA (eSpA) and explore associated demographic, clinical, and radiographic characteristics. Methods: We analysed consecutive eSpA patients referred to the combined gastro-rheumatologic clinic of the University of Rome Tor Vergata. Diagnostic delay was defined as the time interval from the date of first symptoms to the date of diagnosis. Conventional radiography $(\mathrm{CR})$ and magnetic resonance images (MRI) of sacroiliac (SI) joints and spine were performed in axial (ax)SpA patient and examined by two independent radiologists. MRI were assessed for the presence of active/chronic inflammatory lesions, disease activity by ASDAS and inflammatory markers. Statistical analyses were performed using Mann-Whitney, chi square/Fisher tests and covariance analysis (SPSS software).

Results: 190 eSpA patients (124 female, mean age $47.5 \pm 12.8$ years, disease duration $72 \pm 67.4$ months, 73 UC/117 CD; 118 peripheral SpA, 72 axSpA including 44 non radiographic ( $n r)$-axSpA) were evaluated. Axial eSpA patients had a higher prevalence of men sex $(p<0.0001)$, HLA-B27 positivity $(p=0.004)$, uveitis $(p=0.01)$ and pancolitis $(p<0.006)$ compared with peripheral eSpA. AxeSpA patients displayed higher ESR, ASDAS, and VAS pain compared with peripheral $E S p A$ ( $p=0.0006, p=0.001, p=0.019$, respectively). A higher prevalence of csDMARDs was detected in peripheral eSpA compared with axSpA $(p=0.002)$ while treatment with cs and bDMARDs was similar in rad-axSpA and nr-axSpA patients.

Median diagnostic delay in eSpA was 48 months (IQR 6-77) with no difference between axial and peripheral patients. Rad-ax-SpA patients displayed a higher diagnostic delay compared with nr-axSpA (median/IQR 36/17-129 vs 31/10-57 months, $p=0.03$ ). Patients with rad-axSpA were older and with longer disease duration than patients with $n r-a x \operatorname{SpA}(p=0.005$ and $p=0.019)$. Low education status and high rate of employment were found in rad-axSpA compared with nr-ax$\operatorname{SpA}(p=0.003$ and $p=0.03$, respectively).

Rad-axSpA patients with sclerosis, syndesmophytes and bridge at CR had a higher diagnostic delay than those without lesions $(p=0.03, p=0.043, p<0.0001$ Fig. $1 \mathrm{~A}-\mathrm{C}$ ). Men showed a higher prevalence of spine damage lesions than women as sclerosis $(p=0.02)$, squaring $(p=0.0006)$, syndesmophytes $(p=0.0028)$ and bridges $(p=0.007)$. Longer disease duration was detected in patients with radiographic damage as bridge $(p<0.0001)$ and sacroiliitis grade $3(p=0.04)$. On $\mathrm{MRI}, \mathrm{SI}$ bone oedema was associated with reduced diagnostic delay $(p=0.04)$ while bone erosions was associated with higher diagnostic delay $(p=0.002)$ compared with that in patients without these lesions (Fig. 1D-E). Rad-axSpA women had a higher prevalence of $S I$ damage lesions at MRI than men $(p=0.001)$ Patients with psoriasis displayed a higher diagnostic delay compared to those without skin involvement $(p=0.004)$.
A

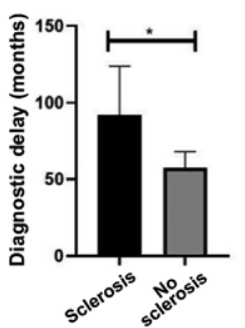

D

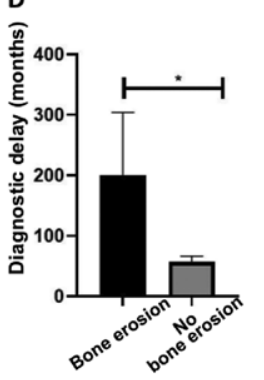

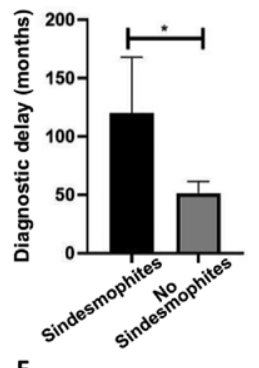

E

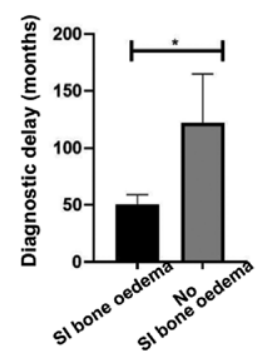

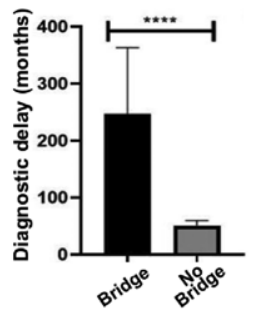

Figure 1. 